\title{
Controlling Soliton Refraction in Optical Lattices
}

\author{
Jaroslaw E. Prilepsky, ${ }^{1, *}$ Stanislav A. Derevyanko, ${ }^{1}$ and Sergey A. Gredeskul ${ }^{1,2}$ \\ ${ }^{1}$ Nonlinearity and Complexity Research Group, Aston University, B4 7ET Birmingham, United Kingdom \\ ${ }^{2}$ Department of Physics, Ben Gurion University of Negev, 84105 Beer Sheva, Israel
}

(Received 26 April 2011; published 17 August 2011)

\begin{abstract}
We show in the framework of the 1D nonlinear Schrödinger equation that the value of the refraction angle of a fundamental soliton beam passing through an optical lattice can be controlled by adjusting either the shape of an individual waveguide or the relative positions of the waveguides. In the case of the shallow refractive index modulation, we develop a general approach for the calculation of the refraction angle change. The shape of a single waveguide crucially affects the refraction direction due to the appearance of a structural form factor in the expression for the density of emitted waves. For a lattice of scatterers, wave-soliton interference inside the lattice leads to the appearance of an additional geometric form factor. As a result, the soliton refraction is more pronounced for the disordered lattices than for the periodic ones.
\end{abstract}

PACS numbers: 42.65.Tg, 05.45.Yv, 42.65.Jx, 42.65.Wi

The applications of the soliton theory are ubiquitous, ranging from nonlinear optics [1] to Bose-Einstein condensation (BEC) [2]. Solitons are highly robust objects which makes them attractive for many practical applications in all-optical devices, information transmission, etc. [1]. One problem drawing considerable recent interest is soliton propagation in a system of spatially localized waveguides [3]. The particular topics involve the study of the formation of nonlinear surface states on interfaces of optically induced [4] or laser-written lattices [5], with the latest studies indicating the specific peculiarities of nonlinear optical transmission in disordered lattices [6,7]. The properties of transverse refractive index modulation for such lattices are nowadays well controllable, which allows one to fabricate a great variety of lattice structures with specially designed individual waveguide profiles (see, e.g., Ref. [8]). Because of the mathematical similarity of the governing equations (which are usually the nonlinear Schrödinger equation and its modifications), many effects predicted for optical solitons have their counterparts in BEC [9], where the influence of both disordered [10] and periodic [11] potential on the correlated matter waves have been addressed recently.

When an optical beam propagating in a nonlinear medium encounters an imbedded optical lattice, it can become trapped, forming localized surface states depending on the initial power [5]. In this Letter, we consider the opposite situation when a fundamental soliton beam does traverse through the lattice and in the process it emits radiation and changes its parameters. Even the presence of a single isolated waveguide (i.e., a scatterer) causes the above effects. Gradually increasing the complexity of the underlying system, in this Letter we first address how the structural form factor (FF), i.e., the particular shape of the defect, influences the angle of soliton refraction and show that this dependence is surprisingly nontrivial.
After that, we consider the properties of soliton transmission through both regular and disordered lattices comprised of the identical waveguides of various shapes. The presence of an optical lattice results in the additional geometric FF, which describes the Bragg scattering of the quasilinear radiation shed by the incident soliton. These emitted and scattered waves interact with each other and with the soliton itself $[12,13]$, so that the interference of the extended modes starts to determine the properties of the localized pulse. This effect can be understood as an effective soliton self-action which can drastically change the transmission characteristics. This change of refraction angle cannot be derived by the "particle" approach of Refs. $[8,14,15]$, since the latter cannot account for wavesoliton interaction at all. The existing studies of transmission through sparse lattices $[13,16,17]$ also do not account for the reciprocal action of the radiation.

We model the pulse propagation by the nonlinear Schrödinger equation for the dimensionless complex amplitude of the beam $u(\zeta, x)[1]$ :

$$
i u_{\zeta}+u_{x x}+2|u|^{2} u=\varepsilon V(x) u .
$$

In the context of nonlinear optics and spatial solitons, $\zeta$ plays the role of the longitudinal coordinate for the beam propagation, and $x$ is the transverse one; in other applications (most notably BEC), the variable $\zeta$ plays the role of time. The change of refraction angle of the beam is exactly equivalent to the change of velocity of soliton matter wave, and in what follows we use the term "velocity" throughout, keeping in mind the one-to-one correspondence between the velocity of a dynamical soliton particle and the refraction angle of an optical beam. Parameter $\varepsilon>0$ is proportional to the depth of the refractive index modulation (or the trap potential in BEC applications) in the transverse direction, the profile of which is given by the effective 
linear repulsive potential $V(x)>0$. As the initial condition, we consider the fundamental soliton of the nonlinear Schrödinger equation incident at the point $x=X$ having the form

$u(\zeta, x)=a \operatorname{sech}[a(x-2 b \zeta-X)] \exp \left[i\left(a^{2}-b^{2}\right) \zeta+i b x\right]$,

where $a$ is the initial soliton amplitude and $2 b$ is its initial velocity. We assume that both coordinates and the field are given in so-called soliton units [1]. The potential $V(x)$ can be either a single localized hump of width $M$ or a sum of identical localized profiles, $v(x)$, forming a lattice.

We start with a specific example (see Fig. 1) of a soliton beam scattering on a single Gaussian-shaped scatterer (inset) and on a regular lattice of 10 such waveguides. The results were obtained by full numerical simulations of Eq. (1). The depth of refraction index was relatively weak, $\varepsilon=0.4$, and one observes that, depending on the initial velocity $2 b$, soliton can either accelerate or decelerate (increase or decrease its refraction angle).

In the case of a regular comb of scatterers, a moving soliton colliding with the lattice produces the backward and forward reflected waves which, when trapped inside the lattice, evolve into spatially oscillating standing patterns. Then there are two effects referring to the change of the direction of soliton motion. First, just after passing the lattice the refracted beam experiences a lateral backward center-of-mass position shift $\Delta X$. This effect (which is the first-order effect in $\varepsilon$ ) has been studied in detail by both the particle approach $[14,15]$ and soliton perturbation theory $[12,13]$, and we will not concentrate on it any further. The

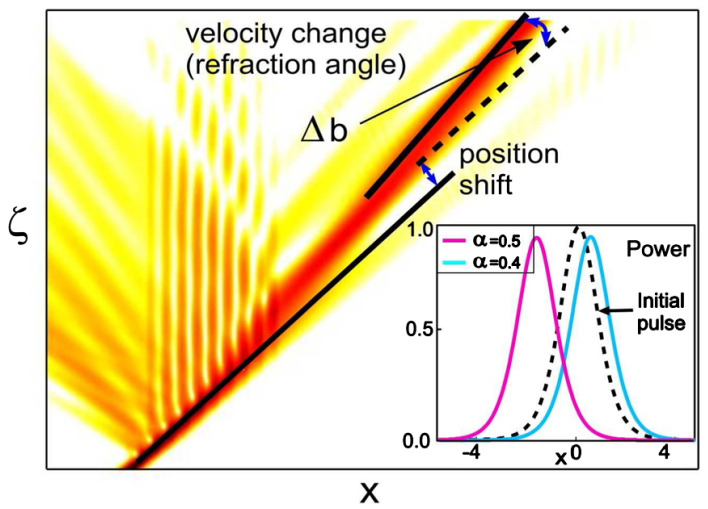

FIG. 1 (color online). The intensity of a soliton beam (log scale) scattered over a periodic comb of identical Gaussianshaped waveguides for $a=1, M=0.2$. The inset shows the profile of a soliton beam scattering on a single defect in the comoving reference frame after a large propagation distance: For the soliton with velocity $2 b=4(\alpha=a / b=0.5)$, the refraction angle decreases (soliton is decelerated, magenta), but for a faster soliton, $2 b=5(\alpha=0.4)$, the opposite effect of acceleration takes place (cyan curve). The initial pulse profile is shown as a dashed line for reference. second visible effect is the correction to the soliton velocity $\Delta b$ (i.e., the change of the refraction angle), which is negative in this particular example. This contribution becomes important at longer distances $\zeta$ from the lattice when the soliton position $X(\zeta)$ is ultimately determined mainly by the velocity change. When the scatterers are stronger, other dynamical regimes can also be observed; see [18].

In the case of shallow index modulation (weak scattering) $\varepsilon \ll 1$, the change of velocity can be described analytically. For the single pointlike scatterer $V(x)=\delta(x)$, the perturbative approach in terms of $\varepsilon$ was developed in Refs. [12,17], where it was demonstrated that in the first order in $\varepsilon$ the velocity does not change at all and $\Delta b=0$ up to terms $\sim \varepsilon^{2}$. It is possible to obtain the second-order changes to the soliton parameters (including the velocity), which was done in Ref. [12]. The key quantity for these calculations is the spectral density of the linear radiation [radiation density (RD)] shed by the soliton after a scattering act. It is defined as the power spectrum of the nonsoliton part of solution, and for an arbitrary decaying scattering potential $V(x)$ the RD can be presented as

$$
n(k)=\frac{\pi \epsilon^{2} \alpha q^{2}}{2^{5} b^{3}} \operatorname{sech}^{2} \frac{\pi(q+a k)}{2 a} F(q), \quad F(q)=|\tilde{V}(q)|^{2},
$$

where $\alpha=a / b, 2 k$ is the wave number, $q(k) \equiv$ $\alpha a\left(k^{2}+1\right) / 2$, and $\tilde{V}(q)$ denotes the Fourier transform of the potential: $\tilde{V}(q)=\int V(x) e^{-i q x} d x[12,17] ; F(q)$ is a corresponding FF. The result above resembles the Born approximation for scattered waves in quantum mechanics. The corresponding second-order correction to the soliton velocity (i.e., the change of refraction angle) is [18]

$$
\Delta b=-\frac{1}{4 a b} \int_{-\infty}^{\infty} d k\left[2 a b k+a^{2}\left(1+k^{2}\right)\right] n(k) .
$$

The sign and the value of the velocity change are determined by two multipliers in the integrand in Eq. (3). The first multiplier in square brackets contains the Doppler term $\sim b k$, and the whole bracket, being negative at small $k$, plays a crucial role in determining the sign of velocity change [the effect is most pronounced when $V(x)$ is a single hump]. The second multiplier in the integrand, $n(k)$, is always positive and defines the measure of this change.

We begin by studying the behavior of $\Delta b$ for singlehump potentials (the case of a single waveguide). The corresponding structural FF and RD for a single shape [Eq. (2)] will be marked with the subscript $S: F_{S}(k)$ and $n_{s}(k)$, respectively. In the limit $V(x)=\delta(x)$, the soliton always decelerates: $\Delta b<0$. However, if the potential has a finite width, the presence of the $\mathrm{FF} F_{S}(k)$ can lead to soliton acceleration after the scattering. In Fig. 2(a), we 
display the velocity change as a function of parameter $\alpha$ for several types of potential. One can see that for a finite-width potential there always exist intervals where the soliton accelerates. Note that there are points where the velocity change turns to zero, so by tuning the parameters of a given profile $V(x)$ one can attain the refractionless transmission (aside from the shift $\Delta X$ ). In Figs. 2(b) and 2(c), we plot the diagram for the sign of the velocity change in the plane of $\alpha$ and width $M$ for the Gaussian and sech potentials. For both shapes, the dependence is qualitatively the same: The slow-heavy soliton (large values of $\alpha$ ) decelerates, while the fast-light soliton (small $\alpha$ ) can also accelerate [red regions in Figs. 2(b) and 2(c)]. The line separating the two regions corresponds to $\Delta b=0$ (the refraction angle does not change). In the limit of zero width, $M=0$, i.e., for the $\delta$ potential, the soliton always decelerates.

In the case of scattering on a lattice, the total RD acquires an additional lattice (geometrical) $\mathrm{FF} F_{L}(k)$, referring to the structure of lattice itself: $n(k)=F_{L}(k) n_{s}(k)$, and the interplay between these two multipliers defines the net lattice effect on the refraction. The FF $F_{L}(k)$ accounts for the resonances of scattered linear waves and has its own minima and maxima. For single-humped scattering profiles, the function $n_{s}(k)$ can have one or two peaks depending on the particular values of parameters $[12,13,17]$, notably $\alpha$. For a given shape of an individual waveguide in the lattice, one can manipulate the waveguide spacing $T$ to adjust the relative positions of the peaks in both factors entering the total RD. Then if the peaks of $F_{L}(k)$ and $n_{s}(k)$ coincide, one enhances the velocity change (refraction angle), whereas in the case of peak mismatch we diminish this change (even cancel it completely in some cases) -
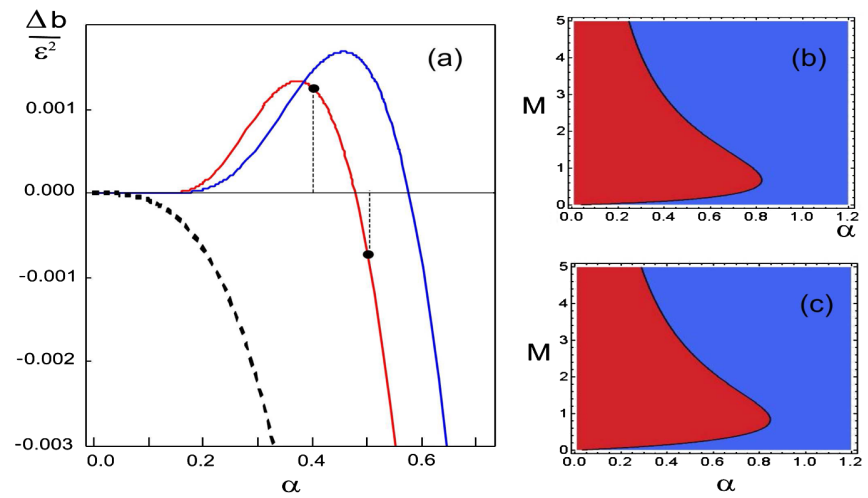

FIG. 2 (color online). (a) Velocity change of a soliton with $a=1$, as a function of $\alpha$ for the Gaussian- (red solid line) and sech- (blue solid line) potential shapes (both with $M=0.2$ ) and the $\delta$ potential (dashed black line). The two dots correspond directly to soliton acceleration and deceleration scenarios shown in the inset in Fig. 1. (b),(c) The phase diagram of the sign of the velocity change for a soliton with $a=1$ for the Gaussian- (b) and sech- (c) scatterer shapes. The red regions correspond to the soliton deceleration and the blue ones to the acceleration. thus controlling the refractive properties of the lattice. This "resonance matching" mechanism is the result of the linearity of the soliton perturbation theory (see [18]).

Consider the lattice of $N$ scatterers with identical profiles $v(x)$, so that $V(x)=\sum_{n=1}^{N} v\left(x-x_{n}\right)$, and start with the case of a regular lattice with the period $T$, so that $x_{n}=n T$. Then the lattice FF is just the Bragg factor [18]:

$$
F_{L}(k)=\sin ^{2} \frac{q(k) N T}{2} / \sin ^{2} \frac{q(k) T}{2}
$$

To minimize the net velocity change, one should suppress the main peak of the $\mathrm{RD} n_{s}(k)$, located, say, at $k=k_{\max }$. It can be done by changing the period of the lattice so that the first Bragg zero of Eq. (4) coincides with $k_{\max }$. Conversely, by tuning the positions of a peak of $F_{L}(k)$ to $k_{\max }$, one can enhance the refraction.

The velocity change as a function of the lattice period for different individual shapes $v(x)$ is given in Fig. 3. In Fig. 3(a), one observes the first matched Bragg zero point producing the minimum of the total refraction followed by a broad plateau corresponding to a larger refraction. The effects are clearly visible for the delta-shaped scatterer and smeared by the structural FF for two other shapes. Higherorder (and much weaker) resonances are also distinguishable. For some values of parameters $\alpha$ and $M$, the density $n_{s}(k)$ has a more pronounced narrow peak so that the magnitude of the refraction dip is higher [Fig. 3(b)].

Let us turn now to the case of random optical lattice formed by a set of $N$ scatterers uniformly distributed within the segment of size $L$, so that the mean distance between adjacent scatterers is $T=L / N$. Intuitively, it is clear that the resonance picture will be smeared, this time owing not just to the structure of the factor $n_{s}(k)$ but because of the higher Bragg resonances of $F_{L}(k)$ being smeared. Indeed, averaging the lattice FF $F_{L}(k)$ for such a model amounts to averaging the sum of independent partial waves with uniform phase differences. The result of this averaging reads [18]
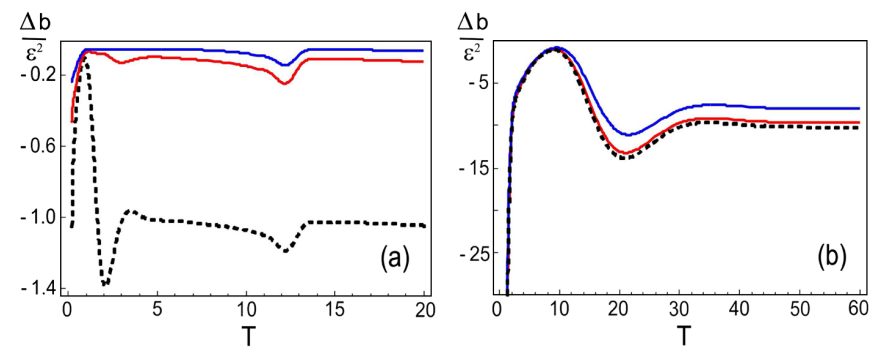

FIG. 3 (color online). The dependence of the velocity change on the period $T$ for the periodic system containing 10 waveguides. Red lines correspond to Gaussian individual shapes; blue lines, to sech shapes, both with width $M=1$ and $a=1$; dashed lines, to $\delta$ scatterers. The parameter $\alpha$ defines the profile of $n_{s}(k)$ : for (a) $\alpha=1$ and (b) $\alpha=0.1$. 


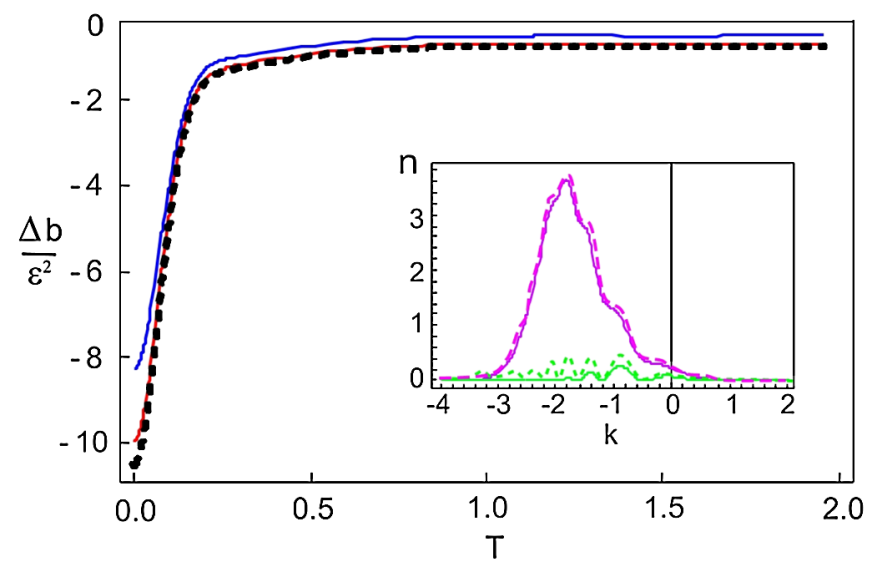

FIG. 4 (color online). The dependence of the average velocity change on $T$ for the random 10-waveguide lattices of $\delta$ (dashed line), Gaussian (blue line), and sech (red line) shapes, $M=0.1$, $\alpha=1, a=1$. The inset shows the dependence of RD $n(k)$ for $T=1$ for the periodic (green line) and random (magenta line) system of 10 Gaussian (solid line) and $\delta$ (dashed line) scatterers.

$$
\left\langle F_{L}(k)\right\rangle=N+\left(1-\frac{1}{N}\right)\left(\frac{\sin [q(k) T N / 2]}{q(k) T / 2}\right)^{2} .
$$

In the case $q T N \ll 1, N \gg 1$, the average FF above displays behavior similar to the periodic lattice: Both FFs scale as $N^{2}$ since the phase shift between the two consecutive scatterers is negligible. However, if the product $q T N$ is not small, the FF of the disordered system approximately equals $N$, while for the regular lattice it varies between zero and $N^{2}$ (the latter correspond to relatively narrow windows near the resonances $\sin q T / 2=0$ ). The inset in Fig. 4 demonstrates the difference between the RDs for random and periodic lattices: In general, a random sequence of waveguides essentially enhances the average amount of the emitted radiation (and hence the velocity change) as compared to its periodic counterpart. In Fig. 4, we plot the dependence of the velocity change for the disordered lattice on the mean distance $T$ : This change is a monotonically increasing function for all profiles. In the limit of very sparse scatterers, it converges to the results of Ref. [13]. A slightly different model of random waveguide array has been studied recently in Ref. [7] in the opposite case of strong scatterers, $\varepsilon \gg 1$. There, however, the authors studied the power reflection coefficients of the pulse power rather than the effects of soliton refraction.

In conclusion, we have studied the influence of both the shape and the positioning of individual waveguides in a modulated optical lattice on the soliton transmission. The case of shallow modulation was described analytically by virtue of perturbation theory in terms of the modulation depth $\varepsilon$. The object of study was the beam refraction angle (velocity). We have developed a general approach for assessing the sign and the measure of the refraction angle for a given configuration of weak scatterers. We have demonstrated that the shape of an individual waveguide has a crucial effect on the direction of the beam refraction angle, and for some values of the waveguide parameters one can suppress the refraction completely. This can be effectively used to control optical refraction by manufacturing individual waveguide profiles in a prescribed way. The waveguide lattice brings about the new effect which accounts for the interference of the emitted waves and the effective soliton self-action. By shifting the relative position of resonant peaks and gaps in both the single-profile $\mathrm{RD} n_{s}(k)$ and the lattice FF $F_{L}(k)$ (by tuning the lattice period), one can effectively control the beam refraction angle. For the disordered system, the average amount of emitted radiation is larger (unless some very special resonance conditions are met) than for the periodic one. The change of the refraction angle for a periodic system has minima and maxima as a function of the lattice period, while for the random scatterers this dependence is monotonically decreasing. Since our work concerns mainly the analytical investigation of the properties of scattering, the validity range of respective results is the same as that of the soliton perturbation theory, i.e., $\varepsilon \ll 1$. However, as additional simulations show [18], the effect of the scatterer shape on the direction of the refraction extends qualitatively even beyond the formal bounds of perturbation theory.

*y.prylepskiy1@aston.ac.uk

[1] Yu.S. Kivshar and G. Agraval, Optical Solitons: From Fibers to Photonics Crystals (Academic, San Diego, 2003).

[2] K. E. Strecket et al., Nature (London) 417, 150 (2002); L. Khaykovich et al., Science 296, 1290 (2002).

[3] J. W. Fleischer, M. Segev, N. K. Efremidis, and D. N. Christodoulides, Nature (London) 422, 147 (2003).

[4] X. Wang et al., Phys. Rev. Lett. 98, 123903 (2007).

[5] A. Szameit et al., Phys. Rev. Lett. 98, 173903 (2007); A. Szameit et al., Opt. Lett. 33, 1132 (2008).

[6] T. Pertsch et al., Phys. Rev. Lett. 93, 053901 (2004); A. Szameit et al., Opt. Lett. 35, 1172 (2010).

[7] Y. V. Kartashov, V. A. Vysloykh, and L. Torner, Opt. Lett. 36, 466 (2011).

[8] Y. V. Kartashov, V. A. Vysloukh, and L. Torner, Prog. Opt. 52, 63 (2009).

[9] O. Morsch and M. Oberthaler, Rev. Mod. Phys. 78, 179 (2006).

[10] J. E. Lye et al., Phys. Rev. Lett. 95, 070401 (2005); C. Fort et al., Phys. Rev. Lett. 95, 170410 (2005); D. Clément et al., Phys. Rev. Lett. 95, 170409 (2005).

[11] Y. Sivan, G. Fibich, and M. I. Weinstein, Phys. Rev. Lett. 97, 193902 (2006).

[12] Yu.S. Kivshar, A.M. Kosevich, and O.A. Chubykalo, Phys. Lett. A 125, 35 (1987).

[13] S. A. Gredeskul, S. A. Derevyanko, A. S. Kovalev, and J. E. Prilepsky, Phys. Rev. E 81, 036608 (2010).

[14] Y. V. Kartashov, A.S. Zelenina, L. Torner, and V. A. Vysloukh, Opt. Lett. 29, 766 (2004); Z. Xu, Y. V. 
Kartashov, and L. Torner, Phys. Rev. Lett. 95, 113901 (2005).

[15] Y. V. Kartashov, V. A. Vysloukh, and L. Torner, Opt. Express 14, 1576 (2006).

[16] Yu.S. Kivshar, S.A. Gredeskul, A. Sanchez, and L. Vazquez, Phys. Rev. Lett. 64, 1693 (1990).
[17] J. C. Bronski, J. Nonlinear Sci. 8, 161 (1998).

[18] See Supplemental Material at http://link.aps.org/ supplemental/10.1103/PhysRevLett.107.083901 for the additional numerical simulations at large $\varepsilon$ and the details of the derivation of Eqs. (3)-(5). 